\title{
UN ESTUDIO SOBRE EL ROZAMIENTO EN LIBROS DE FÍSICA DE NIVEL UNIVERSITARIO
}

\author{
CONCARI, SONIA BEATRIZ ${ }^{1,2}$, POZZO, ROBERTO LUIS ${ }^{1,3}$ y GIORGI, SILVIA MARÍA ${ }^{1}$ \\ ${ }^{1}$ GIDEAF. Grupo de Investigación y Desarrollo en Enseñanza y Aprendizaje de la Física. \\ Departamento de Física. Facultad de Ingeniería Química. Universidad Nacional del Litoral. \\ Santiago del Estero 2829. CP 3000 Santa Fe. Argentina. \\ ${ }^{2}$ Facultad de Formación Docente en Ciencias. Universidad Nacional del Litoral. 9 de julio 2655. \\ CP 3000 Santa Fe. Argentina. \\ ${ }^{3}$ INTEC. Instituto Tecnológico para el Desarrollo de la Industria Química. UNL-CONICET. \\ Güemes 3450. CP 3000 Santa Fe. Argentina.
}

\begin{abstract}
SUMMARY
This work analyses the treatment that is given to friction phenomena in a sample of fifteen physics textbooks for undergraduate level.

From this analysis it emerges that friction phenomena is treated fragmentarily. It is associated -almost exclusivelyeither with dissipating effects or to delaying forces, it is explained using different models for different scales, systems, materials and surfaces conditions, and clear domain limits for empirical laws are not always established, showing inconsistency in the treatment of the subject.

The content analysis technique and linguistic analysis were used in this study. The results are interpreted in the frame of the Rosch prototype theory.
\end{abstract}

\section{INTRODUCCIÓN Y MARCO TEÓRICO}

Existe una gran preocupación por las concepciones que los estudiantes tienen acerca de las fuerzas y el movimiento en general, evidenciada en la extensa bibliografía sobre el tema. En menor medida, recientes estudios ponen la atención acerca de las ideas de los estudiantes sobre las fuerzas de rozamiento (Saltiel, 1994; Islas y Guridi, 1996; Lecumberry, Vicario y Ortiz, 1996) y se formulan propuestas didácticas para su enseñanza (Cotignola et al., 1996, Concari et al., 1995 y Ochoa, 1996). Por el contrario, hay una escasa atención dirigida a cómo es enseñado este tema. Una exploración previa en libros de física básica universitaria permitió detectar algunas cuestiones problemáticas en el tratamiento del rozamiento (Concari et al., 1996).
En los cursos de física básica universitaria, el uso de libros de texto es generalizado. Habitualmente el docente propone la bibliografía y selecciona uno o dos libros como guía para el desarrollo de los distintos tópicos que son objeto de tratamiento en el curso. Estos textos se convierten para él en referentes directos del contenido temático que desarrolla, de los ejemplos que emplea, de los problemas que resuelve y de los problemas que propone a sus alumnos que resuelvan. A través de la participación del alumno en las clases, de la lectura posterior de los apuntes tomados y de la lectura directa del texto, éste se constituye para el estudiante en fuente y guía de estudio. 
Reconociendo que tanto el autor como el lector atribuyen significados a través de la escritura y de la lectura del texto, respectivamente, a fenómenos, conceptos, relaciones y leyes, el presente estudio constituye un análisis realizado sobre el tratamiento que en los textos universitarios se hace del rozamiento. Este tipo de investigación ha comenzado a despertar interés, con relación a la enseñanza de las ciencias, recién en la última década (Laws, 1996), a la luz de estudios recientes que muestran que el material de lectura condiciona fuertemente el aprendizaje (Portolés et al., 1993; Tulip y Cook, 1991).

La enseñanza está siempre relacionada con la comunicación a través del uso de elementos intermedios tales como palabras, textos, dibujos, gestos, símbolos, etc. Es a través de la comunicación que el significado puede ser compartido, con el sentido que le asigna Gowin (1985) al aprendizaje, por el docente y el alumno, por el autor y el lector.

Según afirma Campos Lins (1996): «La producción de significado siempre involucra al menos tres elementos: autor, texto y lector». Los términos autor y lector hacen referencia a quien «enuncia» y a quien «lee» respectivamente el texto, y la noción de texto, en un sentido amplio (no sólo de texto escrito), remite a aquello sobre lo que se produce significado: así se constituye como texto un párrafo de un libro, pero también una ecuación algebraica, una definición formulada oralmente, un diagrama de cuerpo libre, etc.

El autor de un texto asigna significado a lo que enuncia a través del mismo, mientras que el lector produce un significado propio sobre dicho texto que podrá ser más o menos parecido al primero. El modo como el texto es enunciado condiciona la lectura del mismo, su comprensión y, en consecuencia, la posibilidad de construcción de significado. De ahí, entonces, la importancia de la manera en que se formulan los enunciados y se presentan los textos.

El presente estudio se realiza sobre una muestra de libros de física universitaria con el objetivo de interpretar el modo en que son enunciadas las manifestaciones del fenómeno de rozamiento, los modelos explicativos, las leyes empíricas y los ejemplos. El análisis está limitado a libros de texto originalmente escritos en castellano o traducidos a este idioma. En este último caso no se tienen en cuenta posibles deformaciones de los textos originales producto de las traducciones correspondientes.

\section{METODOLOGÍA}

Se empleó como técnica para el estudio, el análisis de contenido (Ander Egg, 1995), a fin de identificar, en el texto escrito, distintas categorías establecidas para clasificarlo. Las categorías de análisis fueron construidas a partir de una exploración previa (Concari et al., 1995), y reformuladas a la luz de las interpretaciones de las lecturas hechas de los textos.
Se presentan a continuación, las siete categorías definidas, así como los valores asignados a las mismas.

Categoría 1: Lugar que ocupa el tratamiento del tema: 1. Como tema de capítulo (Cap). 2. Como subtema de capítulo (SubCap). 3. Como ejemplos y notas complementarias en distintos capítulos (Ej y Not).

Categoría 2: Conceptualización del fenómeno: 1. Como interacción entre sistemas (InSis). 2. Como fuerzas de superficie (FSup).

Categoría 3: Modelos explicativos empleados: 1. Acciones interatómicas o intermoleculares (AIa/Im). 1.1. Teoría de la adherencia (TAdh). 1.2. Colisiones moleculares (ColMol). 1.3. Interacciones electromagnéticas (IEM). 1.4. Vibraciones atómicas (ViAt). 2. Rugosidad de las superficies $(\mathrm{Ru})$. 3. No se explicitan (No).

Categoría 4: Manifestaciones del fenómeno: 1.1. Efecto de frenado traslacional (FrTras). 1.2. Efecto de frenado rotacional (FrRot). 1.3. Efecto disipativo de energía (DisEn). 2.1. Efecto motor traslacional (MoTras). 2.2. Efecto motor rotacional (MoRot).

Categoría 5: Explicitación de leyes empíricas en sistemas: 1.1. Sólido-sólido ${ }^{1}$ (SolSol). 1.2. Sólido-fluido (SolFl). 1.3. Fluido-fluido ( $\mathrm{FlFl})$. 2. No se explicitan (No).

Categoría 6: Límites de validez de las leyes empíricas ${ }^{2}$ : 1. Se explicitan (Si). 2. No se explicitan (No).

Categoría 7: Representación gráfica de las fuerzas de rozamiento: 1.1 . Con vectores sobre esquemas representativos del sistema (Esq). 1.2. Con vectores en diagrama de cuerpo libre (DCL). 2.1. Como única fuerza de interacción superficial (R). 2.2. Como un par de fuerzas $\left(f_{r} y\right)$ ). 3.1. Aplicada en la superficie $\left(f_{r} S u\right)$. 3.2. Aplicada en el centro de masa $\left(f_{r} \mathrm{CM}\right)$. 3.3. Aplicada en otro punto (OtroPto).

La ubicación de las secciones de interés en los textos fue determinada a través de la búsqueda, en el índice general, de las palabras relacionadas directamente con el tema: rozamiento, fricción, frotamiento, fuerza, viscosidad, pérdida de carga. Y de aquéllas que se podían referir indirectamente al rozamiento, tales como: energía, movimiento de traslación y de rotación, fluidos ideales y reales, fuerzas conservativas y no conservativas, etc.

Realizada la lectura del texto correspondiente a las secciones en que éste es tratado, se identificaron en él las ideas relativas a las categorías definidas, codificando las mismas según los valores asignados. Los libros objetos de estudio se listan en el anexo, y serán referidos por su número.

\section{RESULTADOS}

Los resultados se presentan en la tabla I y se analizan a continuación. 
Tabla I

Resumen de los resultados del análisis de los textos.

\begin{tabular}{|c|c|c|c|c|c|c|c|}
\hline Texto & $\begin{array}{c}\text { Lugar del } \\
\text { tratamiento } \\
\text { del tema }\end{array}$ & $\begin{array}{l}\text { Conceptua- } \\
\text { lización } \\
\text { del fenómeno }\end{array}$ & $\begin{array}{c}\text { Modelos } \\
\text { explicativos }\end{array}$ & $\begin{array}{l}\text { Manifesta- } \\
\text { ciones del } \\
\text { fenómeno }\end{array}$ & $\begin{array}{c}\text { Leyes } \\
\text { empíricas } \\
\text { presentadas }\end{array}$ & $\begin{array}{c}\text { Límites } \\
\text { de } \\
\text { validez }\end{array}$ & $\begin{array}{l}\text { Representación } \\
\text { gráfica de la } \mathrm{f}_{\mathrm{r}}\end{array}$ \\
\hline 1 & Cap & FSup & $\mathrm{Ru} ; \mathrm{Adh}$ & FrTRas & SolSol & Sí & $\begin{array}{c}\text { Esq; DCL; R; } \mathrm{f}_{\mathrm{r}} \mathrm{y} \\
\mathrm{N} ; \mathrm{f}_{\mathrm{r}} \mathrm{Su}\end{array}$ \\
\hline 2 & SubCap & InSis; FSup & $\mathrm{AIa} / \mathrm{Im} ; \mathrm{ViAt}$ & FrTRas & SolSol 1 & Sí & Esq; $R ; f_{r}$ y N; $f_{r} S u$ \\
\hline 3 & SubCap; Ej y Not & InSis; FSup & IEM & FrTRas; DisEn & SolSol; SolFl & Sí; Sí & Esq; R; $\mathrm{f}_{\mathrm{r}}$ y N; $\mathrm{f}_{\mathrm{r}} \mathrm{Su}$ \\
\hline 4 & Cap; SubCap & InSis & No & FrTRas; FrRot & SolSol; SolFl & No; Sí & Esq; $\mathrm{f}_{\mathrm{r}}$ y $\mathrm{N} ; \mathrm{f}_{\mathrm{r}} \mathrm{Su}$ \\
\hline 5 & SubCap & FSup & IEM & FrTRas; DisEn & SolSol & Sí & $\begin{array}{l}\text { Esq; DCL; } \mathrm{f}_{\mathrm{r}} \text { y N; } \\
\mathrm{f}_{\mathrm{r}} \mathrm{Su} ; \mathrm{f}_{\mathrm{r}} \mathrm{CM}\end{array}$ \\
\hline 6 & SubCap; Ej y Not & FSup & $\begin{array}{l}\text { AIa/Im(líqu.); } \\
\text { ColMol(gas); } \\
\text { Ru(sólido) }\end{array}$ & $\begin{array}{l}\text { FrTRas; } \\
\text { DisEn }\end{array}$ & $\begin{array}{l}\text { SolSol; FlFl; } \\
\text { SolFl }\end{array}$ & $\begin{array}{l}\text { No; No; } \\
\text { Sí }\end{array}$ & $\begin{array}{c}\text { Esq; DCL; } \mathrm{f}_{\mathrm{r}} \mathrm{y} \mathrm{N} ; \mathrm{f} \\
\text { Su; } \mathrm{f}_{\mathrm{r}} \mathrm{CM} ; \\
\text { OtroPto }\end{array}$ \\
\hline 7 & SubCap; Ej y Not & FSup & No & $\begin{array}{l}\text { FrTRas; } \\
\text { MoRot }\end{array}$ & SolSol; SolFl & Sí; Sí & $\begin{array}{l}\text { Esq; DCL; } \mathrm{f}_{\mathrm{r}} \mathrm{y} \mathrm{N} ; \mathrm{f}_{\mathrm{r}} \\
\mathrm{Su} ; \mathrm{f}_{\mathrm{r}} \mathrm{CM}\end{array}$ \\
\hline 8 & SubCap; Ej y Not & FSup & $\begin{array}{l}\text { Tadh(SolSol); } \\
\text { AIa/Im(FlFl) }\end{array}$ & $\begin{array}{l}\text { FrTRas; } \\
\text { FrRot; } \\
\text { DisEn }\end{array}$ & SolSol & Sí & Esq; $f_{r}$ y $N ; f_{r} S u$ \\
\hline 9 & SubCap & FSup & Tadh; AIa/Im & $\begin{array}{l}\text { FrTRas; } \\
\text { FrRot; } \\
\text { DisEn }\end{array}$ & SolSol; SolFl & Sí; No & $\begin{array}{c}\text { Esq; } \mathrm{f}_{\mathrm{r}} \text { y } \mathrm{N} ; \mathrm{f}_{\mathrm{r}} \mathrm{Su} ; \mathrm{f}_{\mathrm{r}} \\
\mathrm{CM} ; \text { OtroPto }\end{array}$ \\
\hline 10 & SubCap; Ej y Not & FSup & IEM & $\begin{array}{l}\text { FrTRas; } \\
\text { DisEn }\end{array}$ & SolSol & Par & Esq; $f_{r}$ y $N ; f_{r} C M$ \\
\hline 11 & SubCap & FSup & $\mathrm{Ru}$ & $\begin{array}{l}\text { FrTRas; } \\
\text { DisEn }\end{array}$ & $\begin{array}{l}\text { SolSol; FlFl; } \\
\text { SolFl }\end{array}$ & $\begin{array}{l}\text { Sí; Sí; } \\
\text { Par }\end{array}$ & 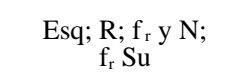 \\
\hline 12 & SubCap; Ej y Not & InSis & IEM; Ru & $\begin{array}{l}\text { FrTRas; } \\
\text { DisEn } \\
\text { MoTras }\end{array}$ & SolSol; FlFl & Par; Sí & $\begin{array}{l}\text { Esq; DCL; } \mathrm{f}_{\mathrm{r}} \text { y N; } \\
\text { OtroPto }\end{array}$ \\
\hline 13 & SubCap; Ej y Not & InSis; FSup & $\mathrm{AIa} / \mathrm{Im}$ & $\begin{array}{l}\text { FrTRas; } \\
\text { FrRot; } \\
\text { DisEn }\end{array}$ & SolSol; SolFl & Sí; Par & $\begin{array}{l}\text { Esq; } \mathrm{f}_{\mathrm{r}} \text { y } \mathrm{N} \\
\text { OtroPto }\end{array}$ \\
\hline 14 & SubCap; Ej y Not & InSis; FSup & $\begin{array}{c}\text { Tadh; AIa/Im; } \\
\text { Ru }\end{array}$ & $\begin{array}{l}\text { FrTRas; } \\
\text { MoTRas; } \\
\text { MoRot }\end{array}$ & SolSol & Sí & $\begin{array}{l}\text { Esq; DCL; } \mathrm{f}_{\mathrm{r}} \text { y N; } \mathrm{f}_{\mathrm{r}} \\
\mathrm{Su} ; \mathrm{f}_{\mathrm{r}} \mathrm{CM} ; \text { OtroPto }\end{array}$ \\
\hline 15 & SubCap; Ej y Not & InSis; FSup & $\begin{array}{c}\text { Tadh; AIa/Im; } \\
\text { Ru }\end{array}$ & $\begin{array}{l}\text { FrTRas; } \\
\text { DisEn; } \\
\text { MoTras }\end{array}$ & SolSol & Sí & $\begin{array}{c}\text { Esq; DCL; } \mathrm{f}_{\mathrm{r}} \\
\text { y N; } \mathrm{f}_{\mathrm{r}} \mathrm{Su} ; \mathrm{f}_{\mathrm{r}} \mathrm{CM} ; \\
\text { OtroPto }\end{array}$ \\
\hline
\end{tabular}

1 No se hace una presentación formal de las leyes, sino una adecuada discusión sobre las mismas y sobre sus límites de validez.

\section{Lugar que ocupa el tratamiento del tema}

En general, el tema está tratado en cada libro en varios capítulos (entre dos y ocho) referidos a dinámica del punto y de sistemas, mecánica de fluidos, trabajo y energía, calor y temperatura. En dos de los textos analizados (1 y 4), se trata el tema en un único capítulo. En el resto, se presentan dos niveles de tratamiento: desarrollado como subtemas de capítulo y mencionado en ejemplos y notas complementarias en otros capítulos. 
En casi todos los textos analizados, el tópico aparece al tratar los movimientos de traslación como una fuerza indeseable que dificulta el movimiento de los cuerpos; se asocia con los movimientos de rodadura, convirtiéndose en un momento que impulsa la rotación, y resurge nuevamente como efecto de viscosidad en el tratamiento de la dinámica de los fluidos.

\section{Conceptualización del fenómeno}

La mayoría de los autores interpretan el fenómeno como fuerzas de interacción entre superficies de sistemas físicos en contacto. No obstante, en algunos casos dicha explicación es ambigua, como, por ejemplo, al explicitar que la fuerza de rozamiento es debida a la «interacción del objeto con el medio que lo rodea» (12, p. 114).

Se identifica el fenómeno definiendo una fuerza de rozamiento, de roce o de fricción indistintamente, excepto en el libro 12 , donde se privilegia el término fricción asociado a disipación de energía, y el término rozamiento cuando se alude a las leyes del movimiento. El término fuerza es empleado en sistemas sólidosólido, mientras que, para sistemas sólido-fluido y fluido-fluido, se priorizan el término de resistencia o el de viscosidad.

\section{Modelos explicativos empleados}

A excepción de los libros 4 y 7 , en el resto de los textos se hace referencia al menos a uno o más modelos explicativos. Aquéllos que explicitan más de un modelo, lo hacen en función de diferentes criterios: dependiendo de la escala (macro o micro), de la naturaleza de los sistemas físicos en interacción (sólido, líquido, gas) y de los materiales (metales, plásticos, etc.).

En el caso de la interacción sólido-sólido, casi la mitad de los textos explican el fenómeno, asociándolo a la rugosidad de las superficies de los sistemas en contacto. Estas ideas son introducidas desde el inicio mismo del desarrollo del tema: «Entre dos superficies sólidas en contacto, existe rozamiento porque incluso la superficie que puede parecer más lisa es rugosa a escala microscópica.» (6, p. 50). «Los coeficientes estático y cinético de rozamiento [son] más o menos grandes si las superficies son rugosas, y pequeños si son pulidas.» $(11$, p. 36). La mayoría de los libros utiliza el modelo de interacción entre moléculas o átomos de los cuerpos en contacto. La teoría de la adherencia es mencionada explícitamente únicamente en cinco de ellos, y se alude al carácter electromagnético de dichas interacciones en otros cuatro (Tabla I). Las vibraciones entre los átomos como posible mecanismo del rozamiento son mencionadas sólo en un texto (2).

\section{Manifestaciones del fenómeno}

La fuerza de rozamiento es asociada en todos los textos a un efecto de frenado traslacional. A título de ejemplo: «la fuerza de frotamiento es de sentido contrario al movimiento.» (4, p. 322). «Esta fuerza es siempre opuesta al movimiento, o sea, opuesta al vector velocidad $v$ $(10$, p. 94). [...] esta fuerza posee siempre la dirección opuesta a la velocidad.» (13, p. 145). «Las fuerzas de fricción siempre se oponen, en forma automática, al movimiento y nunca lo favorecen.» $(9$, p. 110). «La fuerza de rozamiento, que siempre tiene sentido opuesto al del movimiento [...].» (6, p. 50). «La fuerza de rozamiento se opone siempre al movimiento [...].» $(6$, p. 51).

En cuatro de los libros se pone de manifiesto el efecto de frenado al movimiento de rotación, y en diez de ellos se hace referencia al carácter disipativo del fenómeno de fricción. Así, por ejemplo, en el libro 9, en el tratamiento del movimiento oscilatorio amortiguado se dice que «la energía del oscilador es disipada poco a poco por la fricción y tiende al valor cero conforme transcurre el tiempo» (p. 335); y en 13: «Puesto que [...] el trabajo realizado por la fuerza es siempre negativo, así pues, siempre hace disminuir la energía mecánica del sistema.» (p. 430).

En tres textos $(12,14$ y 15$)$ se hace referencia al efecto motor de la fuerza de rozamiento en el movimiento de traslación. En uno se afirma: «En algunos casos, la fuerza de rozamiento es un elemento útil e incluso se favorece [el rozamiento]. Por ejemplo, se esparce arena sobre los pasillos y las carreteras con hielo, a fin de aumentar la tracción entre la superficie y los zapatos o llantas de los vehículos.» (15, p. 135). En forma un poco más explícita y con un esquema, el mismo autor hace referencia a la fuerza de fricción como la «que evita que el pie resbale hacia atrás» $(14$, p.123), y señala que dicha fuerza tiene la dirección del movimiento. No obstante, en un esquema ilustrativo se muestra el par de fuerzas de acción y reacción representadas con vectores ubicados ambiguamente, donde se denota como fuerza de fricción sólo a una de ellas $(f)$, en los textos al pie del esquema (Fig. 1).

El efecto motor rotacional sólo es tratado por 7 y 14 y es expuesto de forma ambigua en 12, en la solución de un problema cuando hace referencia, por primera vez, a una fuerza $F$ (representada en el esquema con un vector dibujado en la parte frontal de un auto) como «fuerza de rozamiento estática que es la que impulsa el auto» (p. 174), mientras que, en el planteo del mismo problema, presenta otra fuerza (indicada en el esquema con un vector $f$ en la parte trasera del auto) como «fuerza de resistencia» que «tiene su significado acostumbrado».

\section{Leyes empíricas presentadas y límites de validez de las mismas}

En todos los libros de la muestra analizada se presentan las leyes empíricas aplicables a sistemas sólido-sólido. Sin embargo, en cinco de ellos, no se alude a los límites de validez de las mismas y, en otros, sólo se hace sin especificarlos. 
Figura 1

Reproducción de (14), página 123.

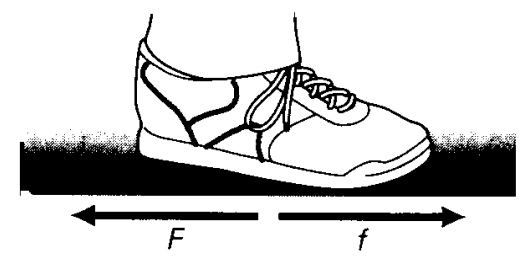

En la mayoría de los textos se aborda el tema a través de los clásicos ejemplos del bloque apoyado sobre una mesa horizontal, complicándolo después con el caso en que la superficie de apoyo está inclinada. La introducción del tema a través de estos ejemplos da como resultado un análisis limitado, transmitiendo implícitamente la idea de fuerza de rozamiento definida por estas leyes.

Las leyes experimentales relativas a los sistemas sólidofluido y fluido-fluido se presentan en nueve y tres de los libros analizados respectivamente, en la mayoría de los casos, en capítulos diferentes.

\section{Representación gráfica de los diagramas}

La fuerza de rozamiento se representa en diagramas esquemáticos, representativos de la situación física que describe el problema, en casi la totalidad de los libros. En cambio, el diagrama de cuerpo libre, entendido como una representación vectorial de las fuerzas aplicadas al sistema físico en estudio, sólo es presentado en menos de la mitad de los textos.

En dichos esquemas o diagramas no aparece la fuerza de interacción entre las superficies en contacto como una única fuerza, sino como dos vectores perpendiculares entre sí, representativos de la fuerza de rozamiento y de la fuerza normal, respectivamente. Como excepción, en el libro 11 y en el 2 se representan, simultáneamente, la fuerza de reacción de la superficie de apoyo y sus componentes aplicados sobre el cuerpo apoyado; y en el libro 1, en diagramas distintos: primero en diagramas con dos componentes y más adelante con una única fuerza de reacción.

Por otro lado, el vector que representa la fuerza de rozamiento en el caso de bloques que deslizan es ambiguamente ubicado en los esquemas en posiciones diversas (incluso en un mismo libro): en la superficie de interacción, en el centro de la masa o, incluso, en algún punto del cuerpo entre el centro de la masa y la superficie, como se ilustra en las figuras 2 y 3, a título de ejemplo.

En general no se tiene mayor cuidado en diferenciar la ubicación correcta de las fuerzas a través de vectores en un esquema representativo de la situación problemática y su tratamiento en un diagrama de cuerpo libre como vectores concurrentes a los efectos de aplicar las ecuaciones de Newton. Una excepción la constituye el libro 14, donde para cada situación se hacen ambas representaciones en forma correcta.

\section{ANÁLISIS DE LOS RESULTADOS}

En el tratamiento del fenómeno de rozamiento, en la mayoría de los textos se encuentra que los fundamentos usados para explicar la fricción surgen de resultados de observaciones experimentales (a escala macroscópica) que datan de alrededor de trescientos años atrás. En el siglo XVII, Amontons explicó el fenómeno de rozamiento a través de las siguientes conclusiones:

1) La fuerza de fricción que se opone al deslizamiento en una interface es proporcional a la «carga normal»o fuerza total que aprieta las superficies.

2) En contra de la intuición, la magnitud de la fuerza de fricción no depende del área de contacto.

En el siglo XVIII, Coulomb completa la explicación expresando:

3) Una vez que ha empezado el movimiento, la fuerza de rozamiento es independiente de la velocidad.

Sin embargo, en los últimos años, la nanotribología ha demostrado que las leyes de la fricción macroscópica no

Figura 2

Reproducción de (12), página 114.

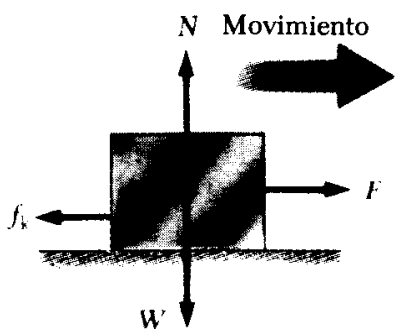

Figura 3

Reproducción de (5), página 120.

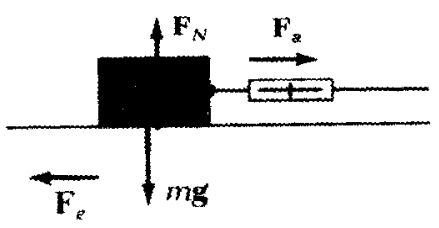


rigen a escala atómica (Krim, 1996). Los estudios más recientes sobre el tema proponen las leyes de rozamiento de una manera más general:

1) La fuerza de fricción depende de la facilidad con la que se queden las dos superficies pegadas en comparación con su facilidad de despegarse: es proporcional al grado de irreversibilidad de la fuerza que aprieta una superficie contra la otra, no a su mera magnitud.

2) La fuerza de fricción es proporcional al área de contacto real, no a la aparente.

3) La fuerza de fricción es directamente proporcional a la velocidad de deslizamiento de la interface en los puntos de contacto reales, siempre y cuando no se deje que las superficies se calienten y mientras la velocidad de deslizamiento esté bastante por debajo de la velocidad del sonido (a velocidad cercana a la del sonido la fricción se estabiliza porque las vibraciones de la red no pueden hacer suya la energía sonora con suficiente rapidez).

Como dice Krim, la inconsistencia entre las leyes de fricción a escala macro y micro se borra si se cae en la cuenta de que es muy probable que el área real de contacto de los objetos macroscópicos sea proporcional a la fuerza que aprieta las superficies. Cuanto más se apriete, habrá mayor superficie de contacto. Por eso, la presión parece ser proporcional a la carga normal, como dijo Amontons en el siglo XVII.

En casi todos los textos analizados, el tópico se presenta como una fuerza indeseable que dificulta el movimiento traslacional, luego como efecto impulsor de la rotación, y se restituye más adelante su condición de fuerza resistente como efecto viscoso. Esta presentación fragmentada del fenómeno de fricción en distintos temas y capítulos, junto con la explicitación de modelos diferentes para distintas escalas, sistemas, materiales y condiciones de las superficies, y con límites de validez de las leyes empíricas no siempre claramente delimitados, evidencia un alto grado de inconsistencia en el tratamiento del tema.

La conceptualización marcadamente intuitiva del fenómeno como derivado de la rugosidad de las superficies está reiteradamente presente. No obstante, su validez está limitada a escala macroscópica y es un modelo desactualizado según los últimos estudios; «su importancia ha quedado en la nada» (Krim, 1996). Es además pedagógicamente inadecuado en la medida en que no favorece el cambio conceptual al haber incoherencia entre las concepciones, las teorías y las evidencias empíricas, pues es fácil demostrar que hay que ejercer una fuerza tangencial intensa para separar dos transparencias cuyas superficies son muy lisas.

Otros modelos mencionados (acción interatómica e intermolecular, teoría de la adherencia, interacción electromagnética, vibración atómica, colisión molecular) involucran elementos de mayor complejidad con relación a la comprensión por parte del estudiante a quien está dirigido el texto. Islas y Guridi (1996) interpretan que «el tránsito del enfoque microscópico al macroscópico parece ser problemático» en los estudiantes. Sería más consistente con el estado del arte en el tema mencionar, como lo hace Feynman (2), que el fenómeno de la fricción aún no se comprende en su totalidad. El tratamiento que este autor hace del tema con relación a la coherencia y consistencia teóricas y empíricas es destacable.

Los alumnos tienen interpretaciones del mundo y dan un sentido a las palabras que ejercen un impacto significativo en su aprendizaje e influyen de forma prácticamente inesperada en el mismo (Osborne, 1991). Dentro de los factores que dificultan la adecuada conceptualización del fenómeno en cuestión podemos identificar el significado que se da en la vida cotidiana a los términos rozamiento, fricción o roce como un obstáculo (o algo) que se opone al movimiento, el cual es reforzado con el tratamiento que en la mayoría de los textos analizados se hace del tema.

En el marco teórico en el que se aborda la fricción (mecánica newtoniana), el tratamiento en los textos es en general incoherente con la tercera ley de Newton, puesto que, como producto de una interacción entre dos sistemas, sobre uno de ellos se representan dos fuerzas (fuerza de rozamiento y normal) en lugar de una como lo predice la mencionada ley, y en ningún caso se explicita la otra fuerza del par acción-reacción actuante sobre el otro sistema a través de la superficie de contacto. Por otro lado, la forma imprecisa, y con los más diversos criterios con que se representan esquemas y diagramas de fuerzas en los libros, no contribuye a que los estudiantes identifiquen pares de acción y reacción, punto de aplicación de las fuerzas y sistema de referencia elegido.

Afirmaciones registradas, por Islas y Guridi, de estudiantes avanzados de profesorado, confirman la incidencia que este tipo de diagramas tiene en relación con la construcción de significado sobre el tema: «Yo tomé los diagramas como aparecen en los libros, cuando marcan las fuerzas. [...] Nunca encontré en un libro la acción y la reacción a la fuerza de roce. Encontré una fuerza que dice $f_{r}$ y que te indica que, si el cuerpo se mueve hacia la izquierda, la fuerza de roce es hacia la derecha. [...] En el caso de la rueda, ahí sí, no va en contra del movimiento [...] Pero no recuerdo si lo he visto en algún libro, ese diagrama, o no.

\section{INTERPRETACIÓN DE LOS RESULTADOS EN EL MARCO DE LA TEORIA DE ROSCH}

En todos los textos, el tema es introducido a través de situaciones como la de un bloque que se pretende mover sobre una superficie de apoyo. Esta situación problemática es exhaustivamente analizada así como variantes de la misma, particularmente la de un bloque apoyado sobre un plano inclinado. Esto no sólo aparece en los libros de texto, sino también en trabajos relacionados con pro- 
puestas didácticas sobre el tema (Wehrbein, 1992; Ochoa, 1996; Cogtignola, 1996; Broitman, 1987).

En aquellos textos en los que se trata el rozamiento sólido-fluido, el ejemplo más empleado es el de un cuerpo que se mueve en el aire, como es el caso de un auto. Casi no se presentan ejemplos de rozamiento fluido-fluido.

Interpretamos que los autores de los textos han hecho un tratamiento del fenómeno de rozamiento sesgado de un «efecto de tipicidad». Por este efecto postulado por Rosch (1978), no todos los ejemplos de una dada categoría serían igualmente representativos de ella. De este modo, existirían ejemplos típicos o «prototipos» y ejemplos de la categoría menos representativos. Los prototipos serían los ejemplos que comparten un mayor número de atributos con otros miembros de la categoría. Estos atributos no son independientes unos de otros, ni son percibidos del mismo modo.

Tomando la teoría del prototipo de Rosch, identificamos como el prototipo de fuerza de rozamiento presentada en los textos aquélla que reúne las siguientes características o atributos:

1) Su existencia se debe a la rugosidad de las superficies de los cuerpos en contacto.

2) Actúa sobre un cuerpo sólido que desliza sobre otro.

3) Ejerce sobre el cuerpo un efecto retardador de su movimiento.

4) Provoca un efecto disipativo de la energía del cuerpo.

5) Su intensidad está definida por la relación: $\mathrm{f}=\mu N^{3}$.

Cuantos más atributos de las listas reúna una fuerza de rozamiento, más prototípica será, siendo el atributo número 3 el detectado preferentemente. Una fuerza, por ejemplo, que sólo reúna los rasgos 1,2 y 5 tendrá menor grado de tipicidad y, en cambio, estará muy lejos del prototipo si no tiene ninguno o muy pocos de esos atributos.

Raramente se presentan en los textos estudiados situaciones problemáticas en las que estén presentes fuerzas de rozamiento de bajo grado de tipicidad, como, por ejemplo, las que actúan sobre los goznes de una puerta, entre dos cubreobjetos o dos transparencias que se quiere separar, sobre el eje de una rueda en movimiento, sobre burbujas que ascienden en el seno de un líquido, sobre las gotas de lluvia en el aire o sobre los objetos arrastrados por una corriente de agua. No se analizan tampoco situaciones cotidianas como la de una persona que camina, la formación de vórtice al agitar un líquido, así como otras que se han propuesto para la enseñanza del tema, en un trabajo anterior (Concari et al., 1995).

El efecto de tipicidad descrito condiciona y limita las representaciones que se construyen de la fuerza de rozamiento. El empleo de ejemplos de fuerzas de rozamiento de alto grado de tipicidad dificulta la comprensión integral de la fricción como un fenómeno de interacción superficial, con efectos diversos y dependientes de las condiciones particulares de la situación. Además, al identificar el fenómeno con aspectos salientes relativos a un tipo particular de fuerza de rozamiento (prototipo), como es, por ejemplo, su efecto retardador o disipativo, se generalizan incorrectamente esas propiedades a cualquier situación.

\section{AGRADECIMIENTO}

Esta investigación ha sido realizada con el apoyo de la Universidad Nacional del Litoral a través de los proyectos CAI+D 94-95 N: 091 y CAI+D 96-97 N: 202.

\section{NOTAS}

${ }^{1}$ Incluye superficies sólidas lubricadas.

2 Para sistemas sólido-sólido, $\mathrm{F}=\mu_{\mathrm{e}} N$ : movimiento incipiente, $\mathrm{F}=\mu_{\mathrm{k}} N$ : independiente de la velocidad; $\mu_{\mathrm{e}} \mathrm{y} \mu_{\mathrm{k}}$ : dependientes de la naturaleza y de las condiciones de las superficies. Para sistemas fluido-fluido, $\mathrm{F}=\eta \mathrm{dv} / \mathrm{dr}$ : fluido newtoniano en régimen laminar. Para sistemas sólido-fluido, $\mathrm{F} \propto \mathrm{V}^{\mathrm{n}}$, con $n=1$ para bajas velocidades y dimensiones del sólido pequeñas $(\operatorname{Re}<1)$ y ley de Stokes para sólido esférico.

3 En esta relación, $\mu$ es el coeficiente de rozamiento estático o cinético y $N$ es la componente normal a la superficie de contacto, de la fuerza de interacción entre cuerpo y superficie de apoyo.

\section{REFERENCIAS BIBLIOGRÁFICAS}

ANDER EGG, E. (1995). Técnicas de investigación social. 24a. ed. Argentina: Lumen.

BROITMAN, E. (1987). Fuerza de rozamiento. Memorias de la $V$ Reunión Nacional de Enseñanza en la Física. Mar del Plata (Argentina).
CAMPOS LINS, R. (1996). Struggling for survival: the production of meaning. Sheffield (Inglaterra): BSRLM Conference.

CONCARI, S., GIORGI, S. y POZZO, R. (1995). La fuerza de rozamiento: ¿efecto retardador o acelerador del movimiento? 
Memorias de la IX Reunión Nacional de Educación en Física (REF IX), Salta (Argentina), pp.

CONCARI, S., POZZO, R., y GIORGI, S. (1996). El rozamiento en libros de física universitaria: un tema que provoca fricción. 81a. Reunión Nacional de la Asociación Física Argentina (AFA). Tandil (Argentina).

COTIGNOLA, M.I., REBORA, G. y PUNTE, G. (1996). Utilización de distintos mediadores instrumentales para el cambio conceptual: tratamiento del rozamiento estático. Memorias del Tercer Simposio de Investigadores en Educación en Física. La Falda (Argentina).

GOWIN, D. (1985). Hacia una teoría de la educación. Aragón. España.

ISLAS, S.M. y GURIDI, V. M. (1996). Exploración sobre fuerzas de roce y su tratamiento didáctico. Memorias del Tercer Simposio de Investigadores en Educación en Física. La Falda (Argentina).

KRIM, J. (1996). Rozamiento a escala atómica. Investigación y Ciencia, diciembre, pp. 46-53

LAWS, P. M. (1996). Undergraduate science education: a review of research. Studies in Science Education, 28, pp. $1-85$

LECUMBERRY, G., VICARIO, J. y ORTIZ, F. (1996). El papel del rozamiento en el aprendizaje de las leyes de movimiento. Memorias del Tercer Simposio de Investigadores en Educación en Física. La Falda (Argentina).

OCHOA (1996). Fuerzas de roce versus principio de acción y reacción. Tercer Simposio de Investigadores en Educación en Física. La Falda (Argentina).

OSBORNE, R. y FRYBERG, P. (1991). El aprendizaje de las ciencias. Implicaciones de la ciencia de los alumnos. Madrid: Narcea, SA de Ediciones.

PORTOLÉS, J.J.S., LÓPEZ, V.S., VIDAL, E. y GÁMEZ, E.V. (1993). Los estudiantes y los textos de ciencias físicas: un estudio sobre su interacción. Caderno Catarinense de Ensino de Física, 10(3), pp. 204-219.

ROSCH, E. (1978). Principles of categorization, en Rosch, E. y Lloyd, B. (eds.). Cognition and categorization. Hillsdale, N.J.: Erlbaum.

SALTIEL, E. (1994). Raisonnements des étudiants sur le frottement cinétique: l'entrainement. Conferencia, II Simposio de Investigación en Educación en Física (SIEF II). Buenos Aires (Argentina).

TULIP, D., y COOK, A. (1991). A Comparison of Author intentions and Student perceptions about Texbook Characteristic. Research in Science Education, 21, pp. 313-319.

WEHRBEIN, W.M. (1992). Frictional forces on an inclined plane. Am. J. Phys., 60(1).

[Artículo recibido en enero de 1997 y aceptado en septiembre de 1998.]

\section{ANEXO}

\section{Libros analizados}

1. Bedford, A. y Fowler, W. (1996). Estática. Mecánica para ingeniería. Delaware (EEUU): Addison-Wesley Iberoamericana.

2. Feynman, R., Leighton, R. y Sands, M. (1987). Física. Vol 1. Delaware (EEUU): Addison-Wesley Iberoamericana.

3. French, A.P. (1978). Mecánica newtoniana, MIT Physics Course. España: Reverté.

4. Galloni, E. y Ruival, H. (1983). Física (mecánica y acústica). Buenos Aires: Nigar SRL.

5. Gettys, W.E., Keller, F. y Skove, M. (1994). Física clásica y moderna. España: McGraw-Hill.

6. Giancoli, D.C. (1985). Física. Principios y aplicaciones I. España: Reverté.

7. Kittel, C., Knight, W. y Ruderman, M. (1991). Mecánica Berkeley Physics Course. Vol. 1, 2a. ed. España: Reverté.

8. McKelvey, J. y Grotch, H. (1980). Física para ciencias e ingeniería. Tomo 1. México: Harla.

9. Resnick, R., Halliday, D. y Krane (1996). 5a. reimpresión de la 3a. edición. Física Vol. 1. México: CECSA.

10. Roederer, J.G. (1963). Mecánica elemental. Buenos Aires: Eudeba.

11. Sears, F., Zemansky M. y Young, H. (1986). Física universitaria. Delaware, EEUU: Addison-Wesley Iberoamericana.

12. Serway, R. (1993). Física I. 3a. ed. (inglés), 2d. ed. (cast.) México: McGraw-Hill.

13. Tipler, P.A. (1978). Física I. España: Reverté.

14. Wilson, J.D. (1990). Física. 2a. ed. México: Prentice Hall Hispanoamericana, SA.

15. Wilson, J.D. (1991). Física con aplicaciones. 2a. ed. México: McGraw-Hill. 